\title{
Acid-sensing ion channel (ASIC) 4 gene: physical mapping, genomic organisation, and evaluation as a candidate for paroxysmal dystonia
}

\author{
Stefan Gründer ${ }^{\star 1}$, Hyun-Soon Geisler ${ }^{1}$, Shirley Rainier ${ }^{2}$ and John K Fink ${ }^{2,3}$
}

${ }^{1}$ Department of Otolaryngology, Research Group of Sensory Physiology, University of Tübingen, Röntgenweg 11, D-72076 Tübingen, Germany; ${ }^{2}$ Department of Neurology, University of Michigan, W. Medical Center Drive, Ann Arbor, Michigan, MI 48109, USA; ${ }^{3}$ Geriatric Research Education and Clinical Center, Ann Arbor Veterans Affairs Medical Center, Ann Arbor, Michigan, MI 48109, USA

Acid-sensing ion channels (ASICs) are protongated $\mathrm{Na}^{+}$channels. They have been implicated with synaptic transmission, pain perception as well as mechanoperception. ASIC4 is the most recent member of this gene family. It shows expression throughout the central nervous system with strongest expression in pituitary gland. ASIC4 is inactive by itself and its function is unknown. Mutations in ion channel subunits, which are homologues of ASICs lead to neurodegeneration in Caenorhabditis elegans. It has, therefore, been speculated that similar mutations in ASICs may be responsible for neurodegeneration in humans. Here, we show that ASIC4 maps to the long arm of chromosome 2 in close proximity to the locus for paroxysmal dystonic choreoathetosis (PDC), a movement disorder with unknown cause. Ion channel genes have been shown to cause several other paroxysmal neurologic disorders and are important candidate genes for PDC. We established the genomic organisation of the ASIC4 gene and screened a PDC pedigree for mutations in the coding region. Although we identified three polymorphisms in the Cterminal part of the ASIC4 protein, these were not present in each affected subject in the PDC kindred we analysed. Therefore, although the ASIC4 gene is physically mapped to the PDC locus, our data indicates that ASIC4 gene mutation is not the cause of PDC. It remains to be established if mutations in ASIC4 or other ASIC subunits may cause neurological disorders. European Journal of Human Genetics (2001) 9, 672-676.

Keywords: acid-sensing ion channel; ASIC; paroxysmal dystonic choreoathetosis; movement disorder; human chromosome 2

\section{Introduction}

Protongated ion channels (ASICs) belong to the $\mathrm{Mec} / \mathrm{ENaC}$ superfamily of amiloride-sensitive $\mathrm{Na}^{+}$channels. ${ }^{1}$ ASICs are ligandgated ion channels and directly activated by a drop of extracellular $\mathrm{pH}$. So far, six different ASICs encoded by four genes have been cloned (ASIC1a, ASIC1b, ASIC2a, ASIC2b, ASIC3, and ASIC4 ${ }^{1-3}$ ), which assemble into multimeric channels. They are expressed in brain as well as in sensory neurones and have been implicated in the

${ }^{*}$ Correspondence: Stefan Gründer, Department of Otolaryngology, Research Group of Sensory Physiology, University of Tübingen, Röntgenweg 11, D-72076 Tübingen, Germany, Tel: +49 707129 84827; Fax: +497071 22917, E-mail: stefan.gruender@uni-tuebingen.de Received 29 March 2001; revised 12 June 2001; accepted 15 June 2001 sensation of pain as well as in modulation of synaptic transmission. Synaptic vesicles are acidic $(\mathrm{pH}$ 5.5) and release of transmitter can, thus, lead to acidic transients in the synaptic cleft. ${ }^{4}$ Therefore, ASICs may contribute to the excitation of neurones.

Mutations in the epithelial $\mathrm{Na}^{+}$channel (ENaC), a homologue of ASICs mediating $\mathrm{Na}^{+}$reabsorption, lead either to channel activation and hypertension or channel inactivation and salt wasting in infancy ${ }^{5-7}$. Mutations in homologues of ASICs from Caenorhabditis elegans cause swelling of neurones and neurodegeneration. ${ }^{8,9}$ These mutations lead to constitutive activation of the channels and constant inflow of cations. ${ }^{10}$ Mutations at the corresponding position can also constitutively activate ASICs ${ }^{11}$ and it has, therefore, been speculated that activating mutations in ASICs may lead to 
similar neurodegeneration in humans. Nonetheless, such mutations have not been reported to date.

The genes for ASIC1 to ASIC3 have already been mapped to the human genome $\mathrm{e}^{12-14}$ but have not been implicated in any hereditary disease. ASIC4 is the most recent member of ASICs. It shows a rather restricted expression pattern with strongest expression in pituitary gland. ${ }^{2}$ But low level of expression is observed throughout the CNS. It is inactive by itself and the physiological role of this channel is unknown. ${ }^{2,3}$

Here we evaluate the potential role of ASIC4 in a neurological disorder. We mapped the gene for ASIC4 to chromosome 2q35-36, in the same region to which the movement disorder paroxysmal dystonic choreoathetosis (PDC; MIM no. 118800) (also known as FPD1, familial paroxysmal choreoathetosis, and PNKD, paroxysmal nonkinesigenic dystonia) ${ }^{15,16}$ was mapped. PDC is characterised by attacks of involuntary movements (dystonia, chorea, and athetosis) occurring spontaneously at rest. They last from minutes to hours and may occur several times each day. Ion channel genes have been shown to cause several other paroxysmal neurologic disorders. ${ }^{17-23}$ Since PDC is an episodic neurologic disorder, ion channel genes are considered important candidates. This prompted the analysis of the ASIC4 gene as a candidate for PDC.

\section{Methods}

\section{Chromosomal localisation}

The human ASIC4 gene was mapped by polymerase chain reaction (PCR) on the GeneBridge 4 radiation hybrid panel (Research Genetics, Huntsville, AL, USA) using primers from the $3^{\prime}$ untranslated region of the ASIC4 cDNA. Sense primer was ASIC4-3' u 5'-CTGGAGACCAGGCCATGGG-3' and antisense primer ASIC4-3' 1 5'-TGGGGCTGGGACCCTGGC-3'. PCR conditions were $94^{\circ} \mathrm{C}$ for $45 \mathrm{~s}, 68^{\circ} \mathrm{C}$ for $45 \mathrm{~s}, 72^{\circ} \mathrm{C}$ for $5 \mathrm{~s}$; 40 cycles. PCR fragments were separated on a $2 \%$ agarose gel and positive samples identified by the appearance of a $209 \mathrm{bp}$ fragment. Results were analysed using the RHMAPPER program of the Whitehead institute (http://carbon.wi.mit. edu:8000/cgibin/contig/rhmapper.pl) with a logarithm of odds (lod)-score of $>21$.

\section{Genomic organisation}

A P1 artificial chromosome (PAC) clone containing the gene for ASIC4 was isolated from the RPCI1, 3-5 library (Roswell Park Cancer Institute, constructed by P Ioannou and P de Jong). Pools of this library (provided by the Resource Center of the German Human Genome Project at the MaxPlanck Institute for Molecular Genetics) were screened by PCR using the same oligonucleotides employed for RHscreening under more stringent conditions $\left(94^{\circ} \mathrm{C}\right.$ for $45 \mathrm{~s}$, $72^{\circ} \mathrm{C}$ for $40 \mathrm{~s} ; 40$ cycles). Maintenance of pools and clones was according to the instructions of the Resource Center. Several positive clones were identified. Clone
RPCIP704F02574 contained the whole gene for ASIC4. The exon/intron structure was established by sequencing. Sequences of exons and adjacent introns were deposited in the EMBL Nucleotide Sequence Database (accession numbers AJ408881 - AJ408884).

\section{Amplification of ASIC4 exons from genomic DNA}

Individual ASIC4 exons and adjacent short intronic sequences were amplified by PCR from human genomic DNA using intronic oligonucleotide primers (See Figure 2). The sequence of these primers and the corresponding PCR conditions are available from the authors upon request. After amplification and agarose gel purification, PCR products were directly sequenced using an ABI377 automated DNA sequencer.

\section{Linkage analysis}

Twopoint linkage analyses were done between ASIC4 codon 508/511 polymorphisms and PDC. We used the Fastlink program, an autosomal dominant model of disease inheritance, and assigned disease allele frequency of 0.001 . We assigned genetic penetrance $=0.90$ for lodscore calculations. We observed two instances of probable incomplete genetic penetrance (subjects IV-2 and IV-3, Figure 4).

\section{Results}

\section{Chromosomal mapping of the ASIC4 gene close to the} PDC locus

We mapped the gene for ASIC4 using the GeneBridge 4 radiation hybrid panel to the human genome. This placed the ASIC4 gene $4.5 \mathrm{cRad}$ (corresponding to approximately $1.2 \mathrm{Mb}$ ) distal of the marker CHLC.GATA3F05 (also known as D2S433) with a lodscore $>21$. This corresponds to position 2q35-36 on the cytogenetic map (Figure 1).

Several diseases have been mapped to this interval, including Bjornstad́s syndrome (MIM no. 262000) and paroxysmal dystonic choreoathetosis (PDC), a movement disorder with unknown cause. It is believed that PDC has its cause in altered metabolism in basal ganglia. ${ }^{24,25}$ Given the expression pattern of ASIC4 with expression throughout the nervous system, we considered the ASIC4 gene as a good candidate for PDC.

We, therefore, analysed a BAC (bacterial artificial chromosome) contig containing the PDC locus for the presence of the ASIC4 gene. The PDC locus, extending from D2S295 to D2S163, is spanned by three BAC contigs (NT_005337, NT_022216 and NT_005289, Genbank). BLAST sequence analysis revealed that the entire ASIC4 gene was contained in two BACs (123-E1 and 158J14) and partially contained in two other BACs (256I23 and 316O14).

Screening of the ASIC4 coding region in a PDC kindred A PAC was isolated containing the entire ASIC4 coding region. The genomic structure of the ASIC4 gene was 
established (Figure 2). Genomic sequences of the genome project, which became available in the public domain during the course of this study, confirm this structure. The whole gene has 10 exons and size of introns ranges from 84 to over $16000 \mathrm{bp}$.

We sequenced the entire ASIC4 coding sequence as well as adjacent intronic sequences (Figure 2) in a DNA sample from an affected member of a large PDC family, in which the disorder had been shown to be linked to the PDC locus on chromosome 2 (D2S295-D2S163; Figure 1). ${ }^{15}$ Since PDC is transmitted as an autosomal dominant disorder, we were

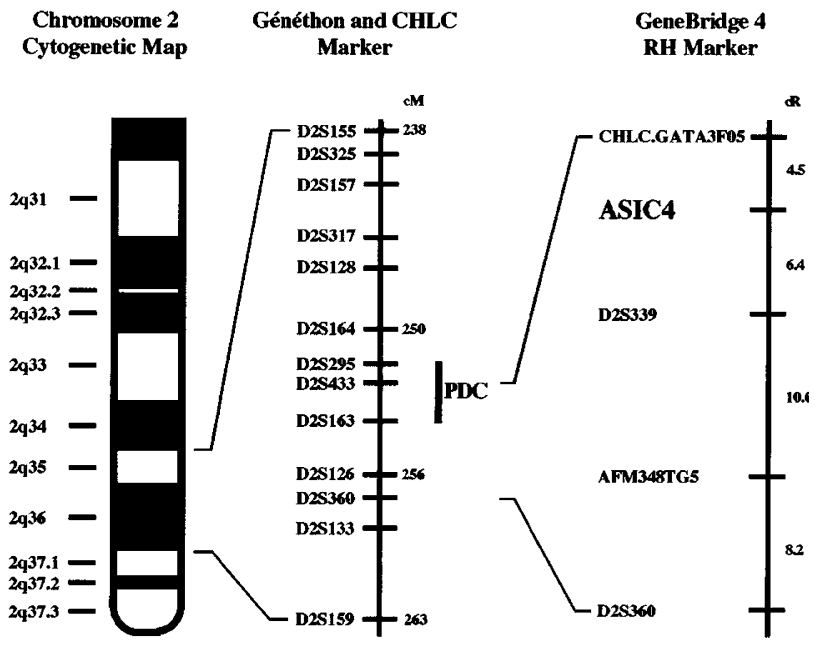

Figure 1 Genomic mapping of the ASIC4 gene. Left, cytogenetic map of the distal part of the long arm of chromosome 2. Center, an enlargement of the 2q35-36 region showing the relative order of Généthon and Cooperative Human Linkage Center (CHLC) markers. Distances are given in cMorgan. The most likely position of the PDC gene between markers D2S295 and D2S163 is indicated. Right, enlargement of the distal part of the PDC interval showing the relative order of markers anchored to the GeneBridge 4 panel. Distances are given in cRad. The most likely position (lod-score $>21$ ) of the ASIC4 gene is $4.5 \mathrm{cR}$ distal to marker CHCL.GATA3F05 (D2S433), placing it in the centre of the PDC interval. particularly interested in identifying heterozygous polymorphisms.

Compared to the human cDNA sequence for $\mathrm{ASIC}^{2}$, we identified one homozygous nucleotide exchange in the first exon, which did not change the amino acid (codon 38 changed from GCC to GCG). In addition, we identified one heterozygous polymorphism in exon 10 changing proline 506 into a glutamine. We refer to this site as the P/Q site. Finally, we identified two homozygous amino acid exchanges, at codon 508 changing leucine into arginine and at codon 511 changing alanine into valine. We refer to these sites as the $\mathrm{L} / \mathrm{R}$ and the $\mathrm{A} / \mathrm{V}$ site, respectively. These three mutations (codon 506 proline to glutamine, codon 508 leucine to arginine, and codon 511 alanine to valine) cluster in a region of only six amino acids contained in the last exon (Figure 3).

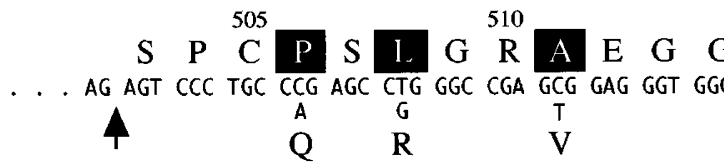

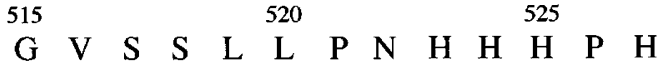
GGG GTC AGC AGT CTG CTC CCC AAT CAC CAC CAC CCC CAC

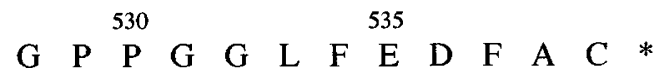
GGT CCC CCA GGa GGT CTC TTT GAA GAT TTT GCT TGC TAG . .

Figure 3 Polymorphisms identified in the last exon of the ASIC4 gene. The last two nucleotides of the ninth intron and the coding region of the tenth exon of the human ASIC4 gene are shown. The arrow indicates the beginning of the exon. Amino acid sequence is shown for human CDNA of ASIC4 ${ }^{2}$. Asterisk indicates the stop codon. Numbering indicates position of the amino acid in the whole protein. Polymorphic sites are highlighted as white letters on black background and amino acids, which were identified in the PDC kindred are shown below the nucleotide sequence; nucleotide as well as resulting amino acid exchanges are shown.

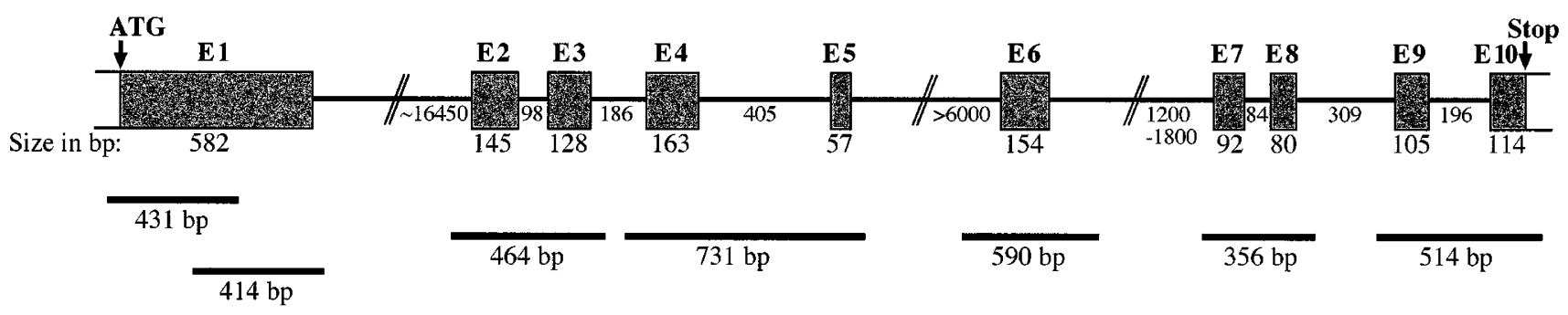

Figure 2 Genomic organisation of the ASIC4 gene. Top, exons are indicated by shaded boxes and introns by lines. The size in base pairs (bp) is given below exons and introns. The position of the start and stop codon is indicated. Size of exon 1 and 10 refers only to the coding sequences. Bottom, PCR-products are indicated by bars, their size is given in bp. 
We then screened the last exon in the other 27 family members for the genotype at these three polymorphic sites. As we did not identify additional polymorphisms of the protein sequence we restricted screening to exon 10 containing these three polymorphisms. Figure 4 summarises the results from this screen. Ten members of the family show clinical symptoms of PDC, the others are not affected. ${ }^{15}$ Only four subjects of the family are heterozygous at the P/Q site, two siblings in generation III and two related inividuals in generation IV (Figure 4). One is an affected person, one is unaffected but passed the disease to her daughter and two are unaffected people. The others are all homozygous for the P. This shows that the P to Q change cannot account for the disease. We identified also individuals heterozygous at the $\mathrm{L} /$ $\mathrm{R}$ and $\mathrm{A} / \mathrm{V}$ sites. The $\mathrm{R}$ was always linked to the $\mathrm{V}$ : the haplotype is L-A on one allele and R-V on the other. As with the $\mathrm{P}$ to $\mathrm{Q}$ change, the $\mathrm{L}$ to $\mathrm{R}$ change and its linked $\mathrm{A}$ to $\mathrm{V}$ change were not disease specific: each was present in both affected and unaffected individuals. Allele frequencies were determined in a cohort of 26 unrelated subjects. P and $\mathrm{Q}$ alleles were $58 \%$ and $42 \%$, respectively; RV and LA alleles were $56 \%$ and $44 \%$, respectively.

Two-point linkage analysis was performed using the syntenic polymorphisms at codons 508 and 511 (subjects determined to be either $508^{\mathrm{Leu}} / 511^{\mathrm{Ala}}$ or $508^{\mathrm{Arg}} / 511^{\mathrm{Val}}$ ) (Figure 4) and assigning genetic penetrance $=0.9$. These polymorphisms were largely uninformative with only several informative subjects. Nonetheless, we obtained a lodscore of +1.68 at $\theta=0$. The absence of obligate recombinants suggests that ASIC4 is linked to PDC and is consistent with the finding that the ASIC4 gene is contained in two BAC clones of the PDC contig (discussed above).

\section{Discussion}

We show that the ASIC4 gene maps to 2q35-36 on the long arm of chromosome 2. PDC has been mapped to this region $^{15,16}$ and the locus reduced to the $2.7 \mathrm{cM}$ interval between D2S295 and D2S163 26,27 (Figure 1). Moreover, ASIC4 was identified as being within a BAC contig containing the PDC disease gene, showing that ASIC4 maps within the PDC locus on chromosome 2.

We identified three amino acid substituting ASIC4 gene polymorphisms in the initial subject, for whom we sequenced the entire ASIC4 coding region. One was a heterozygous mutation (changing proline to glutamine at codon 506) and the other two were homozygous mutations (at codons 508 and 511). Since PDC is dominantly inherited,

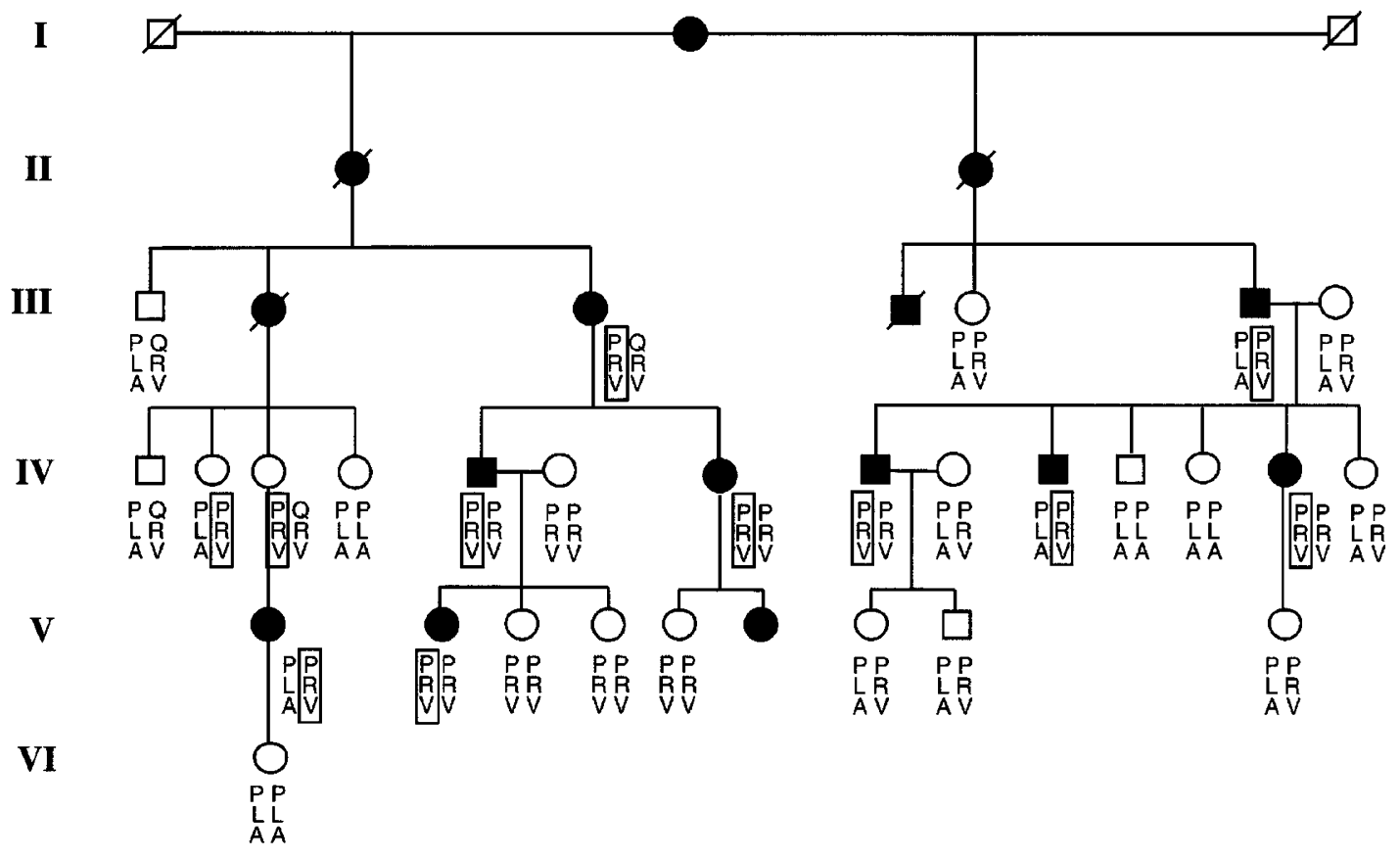

․ O Unaffected male, female

口. Affected male, female

$\square$ Disease alleles are enclosed by boxes.

Figure 4 Segregation of ASIC4 coding sequence polymorphisms with paroxysmal dystonic choreoathetosis. Genotypes are shown for polymorphisms at codon 506 (proline/glutamine), codon 508 (arginine/leucine), and codon 511 (alanine/valine). Disease alleles are enclosed by boxes. Polymorphisms have initially been identified in subject III-3. 
the heterozygous mutation at codon 506 was considered the more likely candidate to account for the PDC phenotype. None of these polymorphisms was disease specific: both wildtype and mutant alleles were found in affected and unaffected subjects. Moreover, we identified these polymorphisms also in unrelated subjects not showing any symptoms of PDC, suggesting that these are rather common polymorphisms. Our data does not support a role for ASIC4 coding sequence mutations in PDC.

Sequence conservation between human and rat ASIC4 is high; both proteins are $97 \%$ identical. $^{2}$ The three polymorphisms we identified map to a region, which shows comparatively high sequence divergence. This may indicate that these polymorphisms do not interfere with normal channel activity.

We show that although ASIC4 maps within the PDC locus on chromosome 2, we did not identify disease (PDC) specific ASIC4 coding sequence mutations. Consensus sites for splicing at the borders of introns were also identical between affected and unaffected family members. We cannot exclude, however, that mutations in other noncoding portions of ASIC4 could contribute to PDC. Closer evaluation of ASIC4 and other ASIC genes will show if mutations in ASICs lead to neurodegeneration or other neurological disorders.

\section{Acknowledgements}

We thank Dr M Pfister for help with the population frequencies. This work has been supported by a grant of the Attempto research group programme of the Universitätsklinikum Tübingen (FG 1-0-0) to $S$ Gründer and by grants from the National Institutes of Health (NINDS R01NS33645, R01NS36177 and R01NS38713) and Department of Veterans Affairs (Merit Review) to JK Fink.

\section{References}

1 Waldmann R, Lazdunski M: $\mathrm{H}(+)$-gated cation channels: neuronal acid sensors in the NaC/DEG family of ion channels. Curr Opin Neurobiol 1998; 8: $418-424$.

2 Gründer S, Geissler HS, Bässler EL, Ruppersberg JP: A new member of acid-sensing ion channels from pituitary gland. Neuroreport 2000; 11: 1607-1611.

3 Akopian AN, Chen CC, Ding Y, Cesare P, Wood JN: A new member of the acid-sensing ion channel family. Neuroreport 2000; 11: $2217-2222$.

4 Krishtal OA, Osipchuk YV, Shelest TN, Smirnoff SV: Rapid extracellular $\mathrm{pH}$ transients related to synaptic transmission in rat hippocampal slices. Brain Res 1987; 436: 352-356.

5 Hanson JH, Schild L, Lu Y et al: A de novo missense mutation of the beta subunit of the epithelial sodium channel causes hypertension and Liddle syndrome, identifying a proline-rich segment critical for regulation of channel activity. Proc Natl Acad Sci USA 1995; 92: 11495 - 11499.

6 Chang SS, Gründer S, Hanukoglu A et al: Mutations in subunits of the epithelial sodium channel cause salt wasting with hyperkalaemic acidosis, pseudohypoaldosteronism type 1 . Nat Genet 1996; 12: 248-253.

7 Gründer S: Liddle's syndrome and pseudohypoaldosteronism type I; in Lehmann-Horn F, Jurkat-Rott K (eds): Channelopathies. Amsterdam, 2000, pp. 277-297.
8 Chalfie M, Wolinsky E: The identification and suppression of inherited neurodegeneration in Caenorhabditis elegans. Nature 1990; 345: 410-416.

9 Driscoll M, Chalfie M: The mec-4 gene is a member of a family of Caenorhabditis elegans genes that can mutate to induce neuronal degeneration. Nature 1991; 349: 588-593.

10 Garcia-Anoveros J, Garcia JA, Liu JD, Corey DP: The nematode degenerin UNC-105 forms ion channels that are activated by degeneration- or hypercontraction-causing mutations. Neuron 1998; 20: $1231-1241$.

11 Waldmann R, Champigny G, Voilley N, Lauritzen I, Lazdunski $\mathrm{M}$ : The mammalian degenerin MDEG, an amiloride-sensitive cation channel activated by mutations causing neurodegeneration in Caenorhabditis elegans. J Biol Chem 1996; 271: 10433 10436.

12 Garcia-Anoveros J, Derfler B, Neville-Golden J, Hyman BT, Corey DP: $\mathrm{BNaC} 1$ and $\mathrm{BNaC} 2$ constitute a new family of human neuronal sodium channels related to degenerins and epithelial sodium channels. Proc Natl Acad Sci USA 1997; 94: 1459-1464.

13 Waldmann R, Voilley N, Mattei MG, Lazdunski M: The human degenerin MDEG, an amiloride-sensitive neuronal cation channel, is localized on chromosome 17q11.2-17q12 close to the microsatellite D17S798. Genomics 1996; 37: 269-270.

14 de Weille JR, Bassilana F, Lazdunski M, Waldmann R: Identification, functional expression and chromosomal localisation of a sustained human proton-gated cation channel. FEBS Lett 1998; 433: $257-260$.

15 Fink JK, Rainer S, Wilkowski J et al: Paroxysmal dystonic choreoathetosis: tight linkage to chromosome 2q. Am J Hum Genet 1996; 59: 140-145.

16 Fouad GT, Servidei S, Durcan S, Bertini E, Ptacek LJ: A gene for familial paroxysmal dyskinesia (FPD1) maps to chromosome 2q. Am J Hum Genet 1996; 59: 135 - 139.

17 Browne DL, Gancher ST, Nutt JG et al: Episodic ataxia/ myokymia syndrome is associated with point mutations in the human potassium channel gene, KCNA1. Nat Genet 1994; 8: $136-140$.

18 Biervert C, Schroeder BC, Kubisch C et al: A potassium channel mutation in neonatal human epilepsy. Science 1998; 279: 403 406.

19 Wallace RH, Wang DW, Singh R et al: Febrile seizures and generalized epilepsy associated with a mutation in the $\mathrm{Na}+$ channel beta1 subunit gene SCN1B. Nat Genet 1998; 19: $366-$ 370 .

20 Ptacek LJ, George AL, Griggs RC et al: Identification of a mutation in the gene causing hyperkalemic periodic paralysis. Cell 1991; 67: 1021-1027.

21 Ptacek LJ, Tawil R, Griggs RC et al: Dihydropyridine receptor mutations cause hypokalemic periodic paralysis. Cell 1994; 77: $863-868$

22 Ptacek LJ, George AL, Barchi RL et al: Mutations in an S4 segment of the adult skeletal muscle sodium channel cause paramyotonia congenita. Neuron 1992; 8: 891-897.

23 Sprunger LK, Escayg A, Tallaksen-Greene S, Albin RL, Meisler $\mathrm{MH}$ : Dystonia associated with mutation of the neuronal sodium channel Scn8a and identification of the modifier locus Scnm1 on mouse chromosome 3. Hum Mol Genet 1999; 8: 471-479.

24 Fink JK, Hedera P, Mathay JG, Albin RL: Paroxysmal dystonic choreoathetosis linked to chromosome 2q: clinical analysis and proposed pathophysiology. Neurology 1997; 49: 177-183.

25 Bohnen NI, Albin RL, Frey KA, Fink JK: (+)-alpha-[11C]Dihydrotetrabenazine PET imaging in familial paroxysmal dystonic choreoathetosis. Neurology 1999; 52: $1067-1069$.

26 Jarman PR, Davis MB, Marsden CB, Wood NW: Genetic linkage and candidate gene studies in a British family with paroxysmal distonic choreoathetosis. Neurology 1997; 48: A996.

27 Hofele K, Benecke R, Auburger G: Gene locus FPD1 of the dystonic Mount-Reback type of autosomal-dominant paroxysmal choreoathetosis. Neurology 1997; 49: 1252-1257. 